\title{
Clarifying the pharmacological mechanisms of action of Shenfu Decoction on cardiovascular diseases using a network pharmacology approach
}

\author{
Qingfeng Zhao ${ }^{1}$, Michihisa Tohda ${ }^{1,2, *}$ \\ ${ }^{1}$ Field of Consilienceology for Wakan-yaku, Major of Biological Information System Course, Graduate School of Innovative Life Science, \\ University of Toyama, Toyama, Japan; \\ ${ }^{2}$ Laboratory of Consilienceology for Wakan-yaku, Section of Neuromedical Science, Institute of Natural Medicine, University of Toyama, \\ Toyama, Japan.
}

SUMMARY Since the molecular mechanisms underlying in the pathogenesis of cardiovascular diseases (CVD) are extremely complex and have not yet been elucidated in detail, CVD remain the leading cause of death worldwide. Traditional Chinese medicine involves the treatment of disease from an overall perspective, and its therapeutic effects on CVD have been demonstrated. However, the mechanisms contributing to the multiscale treatment of cardiovascular diseases at the systematic level remain unclear. Network pharmacology methods and a gene chip data analysis were integrated and applied in the present study, which was conducted to investigate the potential target genes and related pathways of Shenfu Decoction (SFD) for the treatment of myocardial injury. The gene chip analysis was initially performed, followed by network pharmacology to identify differentially expressed genes (DEG) and a functional enrichment analysis. Protein-protein networks were constructed and a module analysis was conducted. A network analysis was used to identify the target genes of SFD. Regarding the results obtained, 1134 DEG were identified using the STRING website. The module analysis revealed that nine hub genes exhibited ubiquitin-protein ligase activity. Therefore, SFD significantly alters the expression of ubiquitination-related genes and, thus, plays an important therapeutic role in the treatment of heart failure. In conclusion, hub genes may provide a more detailed understanding of the molecular mechanisms of action of as well as candidate targets for SFD therapy.

Keywords Cardiovascular diseases, traditional Chinese medicine, Shenfu Decoction, network pharmacology

\section{Introduction}

Cardiovascular diseases (CVD) are the leading cause of death worldwide (1). The application of traditional Chinese medicine (TCM) to the treatment of COVID-19 has been examined and it is now used in clinical settings in China; however, there is currently insufficient evidence for its global expansion (2). The use Chinese herbs to prevent various diseases, including CVD, has been investigated (3). Bioinformatics has become an important tool in the medical field because it effectively reveals relevant knowledge hidden in big data, thereby promoting the discovery of integrated information (4). The core concepts of TCM and network pharmacology are similar (5), with multicomponent therapy and network targets as the basis for TCM (6) and a molecular network for network pharmacology (7). Although many methods may be applied in investigations of the mechanisms of action of TCM in the treatment of CVD, network pharmacology is widely accepted and used because it provides a broad perspective (8).

The cardioprotective effects of Shenfu Decoction (SFD) have been demonstrated. It has been shown to inhibit myocardial cell apoptosis in rats with heart failure (9) and protect against myocardial ischemiareperfusion injury (10). Metabonomics (11) and proteomics also revealed its protective effects against heart failure. Moreover, the chemome (12) and serum pharmacochemistry (13) of SFD have been characterized.

The rapid development of network pharmacology has contributed to a more detailed understanding of and insights into the mechanisms by which TCM 
exerts its effects on complex diseases (14). Therefore, in the present study, network pharmacology was used to elucidate the complex molecular mechanisms of action of SFD in the treatment of CVD. We initially treated cells with the corresponding drugs and then subjected samples to a gene chip analysis. Potential targets were mapped to the corresponding database for a bioinformatic analysis, which provides a bridge for clarifying the mechanisms by which SFD exerts its cardiovascular protective effects.

\section{Materials and Methods}

\subsection{Preparation of the SFD extract}

Two Chinese herbal medicines, ginseng and aconite, were selected at a ratio of 2:1 to prepare SFD. Fortyfive grams of Chinese herbal medicine was added to $900 \mathrm{~mL}$ of water to prepare SFD. The hot water extract obtained was then freeze-dried. Tochimoto Tenkai-do (Osaka, Japan) provided Panax ginseng CA Meyer (Lot No. 008607037) and Aconitum carmichaeli Debeaux (Lot No. 032017001) for the present study.

\subsection{Cell culture and treatment}

The rat cardiac myoblast cell line H9C2 was purchased from the European Collection of Authenticated Cell Cultures (ECACC: Salisbury, England). According to the information provided in the instructions, the medium selected was complete DMEM containing $10 \%$ fetal bovine serum and culture conditions were $37^{\circ} \mathrm{C}$ in a $10 \% \mathrm{CO}_{2}$ humidification box. Three groups were included in the present study: 1) Control group; 2) Model group; 3) SFD treatment group. Doxorubicin (DXR: Sandoz, Yamagata, Japan: Lot No. HY8542)mediated H9C2 cytotoxicity was induced as previously described (15). Following a treatment with SFD or vehicle for 24 hours, cells were incubated with or without DXR $(2 \mu \mathrm{M})$ for another $24 \mathrm{~h}$ to induce cardiotoxicity.

\subsection{RNA extraction}

Total RNA was extracted from H9C2 cells using TRIsure (Bioline, Luckenwale, Germany), and genomic DNA was removed by DNase I (TaKaRa, Ohtsu, Japan), according to the manufacturers' instructions. The quantity and quality of RNA were assessed using a 2100 biological analyzer (Agilent, CA, USA). Highquality RNA samples $\left(\mathrm{OD}_{260 / 280}=1.8-2.0 \mathrm{RIN} \geq 7\right)$ were used to construct sequencing libraries.

\subsection{Microarray data}

Three samples each from the Control, Model, and SFD treatment groups were hybridized with an SurePrint
G3 Mouse Gene Expression v2 $8 \times 60 \mathrm{~K}$ Microarray at $65^{\circ} \mathrm{C}$ for $14 \mathrm{~h}$ to analyze expression levels using microarray methods.

2.5. Identification of differentially expressed genes (DEG)

DEG among Control, Model, and SFD treatment group samples were screened using Venn diagrams. $|\log 2 \mathrm{FC}| \geq$ 1 was considered to be significant.

\subsection{Protein-protein interaction (PPI) network construction and a module analysis}

As a classic PPI network construction tool, the STRING database (16) was employed in the present study, and the standard for significance was a combined score $>$ 0.4. In the module analysis after network construction, Cytoscape's plug-in Molecular Complex Detection (MCODE) was selected (17). The selection of key hub genes relied on the following three characteristics: "degree", "intermediateness", and "intimacy". The criterion selected in the present study was that these three characteristics were higher than the corresponding median values.

\subsection{Enrichment analyses of candidate genes}

WebGestalt is a functional enrichment analysis web tool (18). The WebGestalt online database, as a popular biological analysis database, facilitates analyses of the functions of DEG. The standard for statistical selection is $p<0.05$. WebGestalt's over-representation enrichment analysis method is used to identify and analyze potential targets. As a gene annotation tool, a gene ontology analysis involves a functional analysis that includes molecular function, biological pathway, and cell component analyses (19). The Kyoto Encyclopedia of Genes and Genomes (KEGG) is a well-known path analysis tool (20).

\subsection{Co-expression analysis}

GeneMANIA is a user friendly and flexible web server that is used to generate hypotheses about gene functions, analyze gene lists, and assess gene priority for a functional analysis (21). The database comprises many functions, such as a physical interaction analysis, co-expression predictions, co-localization, and a genetic interaction analysis. These functions were used in the present study to construct the SFD gene network.

\section{Results and Discussion}

\subsection{Identification of DEG}

Gene expression datasets were obtained [SET01: 
Control group vs. Model group, SET02: Model group vs SFD treatment group]. After standardizing microarray results, DEG were identified. The overlap between the two data sets contained 1,134 genes, as shown in the Venn diagram (Figure 1).

3.2. Prediction analysis of pharmacological mechanisms based on network pharmacology and module identification

Based on the STRING database, a PPI network was constructed with a combined score $>0.4$ (Figure 2A), with 908 nodes and 1822 edges together forming a large

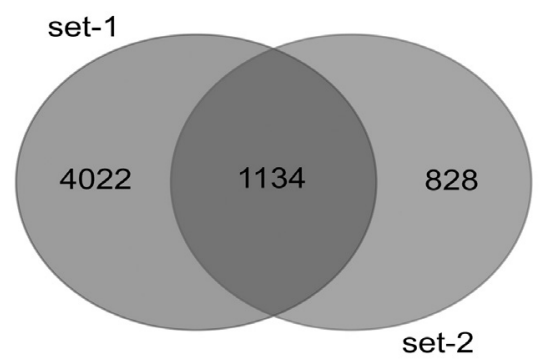

Figure 1. Venn diagram of the number of genes showing treatmentinduced changes in expression. Differentially expressed genes with a fold change $\geq 2$ in microarray data were selected among mRNA expression profiling set-1 (control vs. DXR) and set-2 (DXR vs. SFD + DXR). The two data sets show the overlap of 1,134 genes. biological network (Table S1, http://www.ddtjournal. com/action/getSupplementalData.php?ID=79). The network module (cluster or community) is the subnetwork that is generally defined. The characteristic of the sub-network is that connections between its nodes are more reliable and firmer than the loose connections in the remainder of the network. In other words, a module is a collection of many nodes with a high degree of correlation. Module identification is important because the amount of crucial information extracted from the network is limited, and it facilitates the discovery of vital information by researchers that may be hidden in the network. Network modules have been utilized in network pharmacology-based TCM studies to reveal the combination rule of TCM herbal formulae (22), chemical modules with similar structures (23), and proteins with equivalent functions (24). Multi-component and multi-objective analyses have always been a difficult and hot issue in TCM research. Network modularity is based on the "Law of Similarity Attraction" analysis method, which is a precise and powerful method to describe the complexity of TCM. Figure 2B shows the PPI network of the 293 nodes and 1083 edges obtained with a degree of between 5 and 42 inclusive. Although the scope of Figure 2B is smaller than that of Figure 2A, it is also difficult to identify target molecules. Therefore, as shown in Figure 2C, we established a novel network to identify target molecules

\section{(A)}

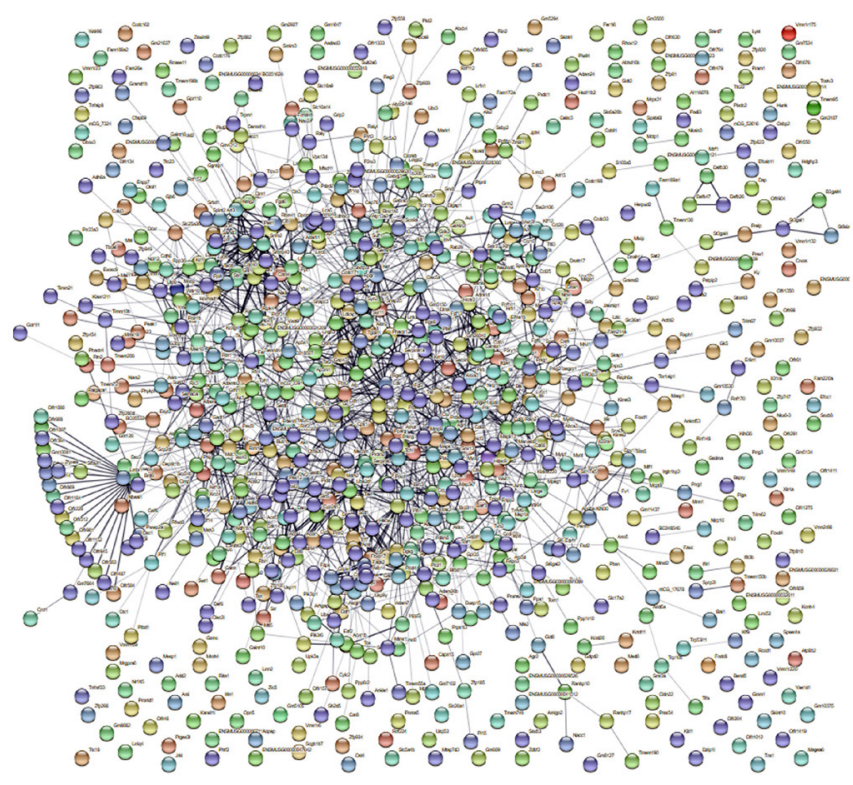

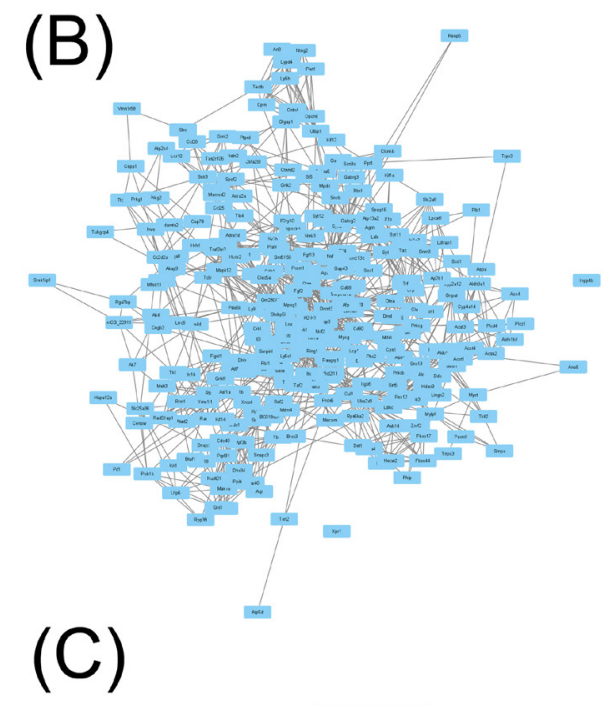

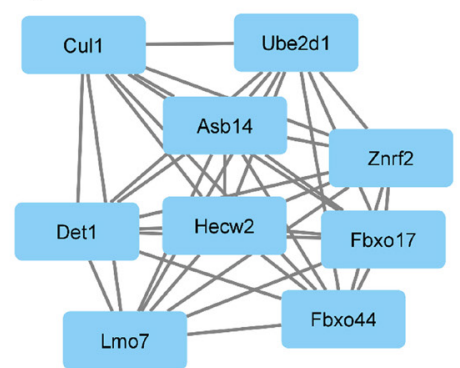

Figure 2. PPI network structure and module identification. (A) PPI network of differentially expressed genes (DEG). (B) The PPI network of DEG was constructed using Cytoscape. (C) The most significant module with 9 nodes and 36 edges was obtained from the PPI network. 
(A)

Bar chart of Biological Process categories

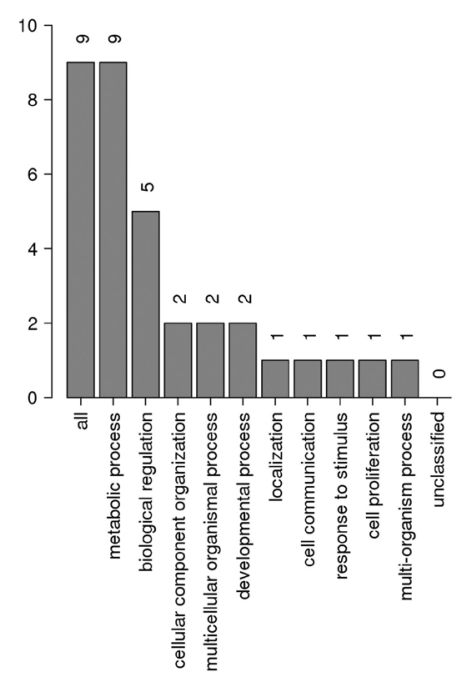

Bar chart of Cellular Component categories

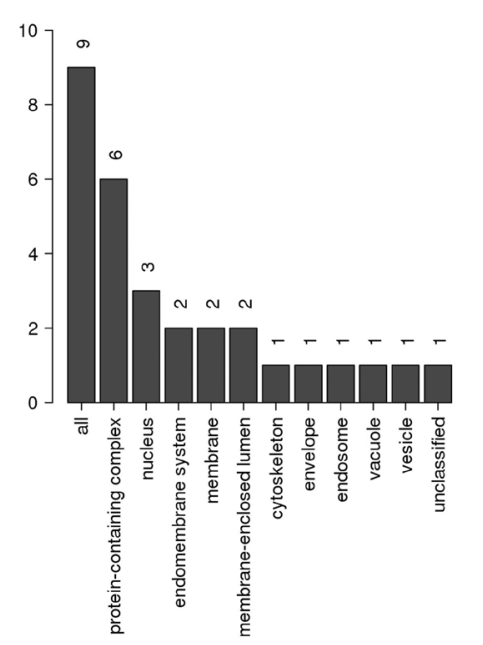

Bar chart of Molecular Function categories

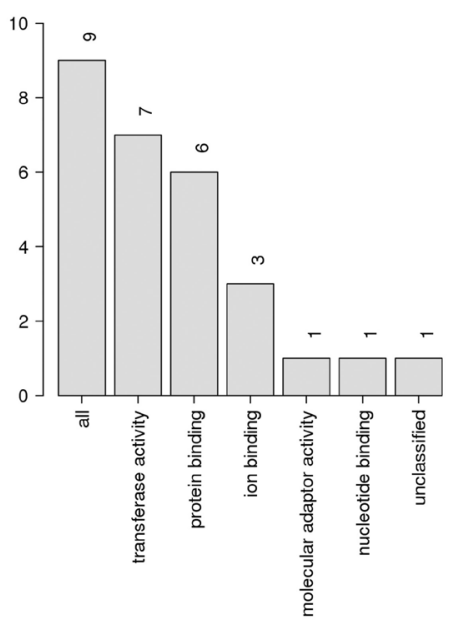

(B)

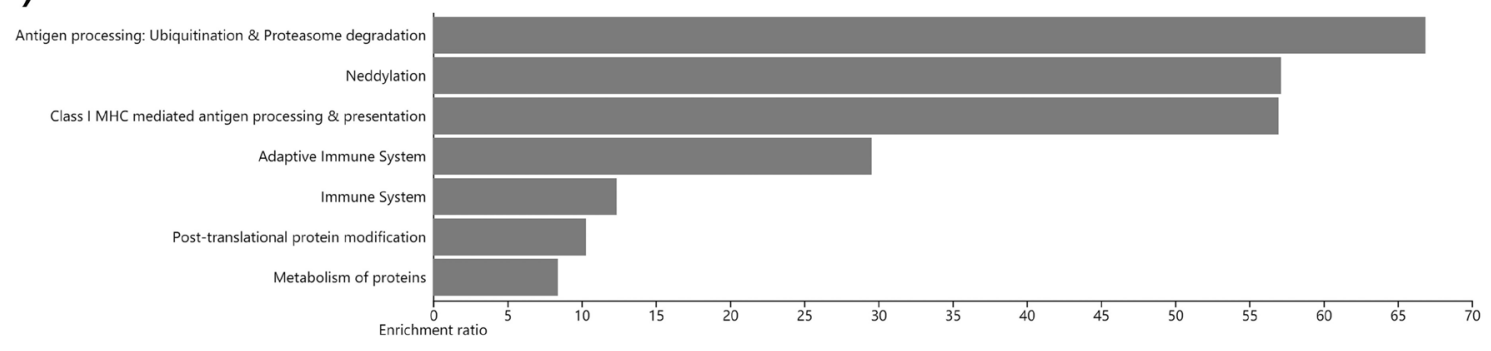

Figure 3. Enrichment analyses of nine hub genes. (A) An enrichment analysis through the WebGestalt database. (B) A KEGG pathway analysis of nine hub genes.

more accurately.

Through the plug-in MCODE, the most significant module was obtained from the PPI network with 9 nodes -36 edges. The names, abbreviations, and functions of these hub genes are shown in Table S2 (http:// www.ddtjournal.com/action/getSupplementalData. php? ID=80). We then calculated three topological features ("degree", "intermediateness", and "compactness") of the candidate molecules. According to the screening criteria described above, three candidate molecules were identified ("degree" > 9, "betweenness" $>0.000111031$, and "closeness" > 0.27027027), as shown in Table S3 (http://www.ddtjournal.com/action/ getSupplementalData.php?ID=80). All 9 genes were associated with the functions of the ubiquitin-proteasome system (UPS), which is important in the etiology of CVD.

UPS plays an essential role in the many mechanisms involved in mediating the degradation of intracellular proteins (25). Ubiquitination and proteasome-mediated degradation are the two main steps of UPS-mediated proteolysis. In other words, ubiquitination is a series of enzymatic reactions in cells (26). Dysfunctional UPS has been implicated in the development of many
CVD (such as atherosclerosis, myocardial ischemia, hypertrophy, and heart failure) (27).

One of the main factors restricting the use of anthracycline antibiotics (such as DXR) in clinical settings is cardiotoxicity (28). DXR-induced cardiotoxicity may increase the activity of UPS (29). Sishi et al. also demonstrated that chymotrypsin-like activity in the heart was inhibited by DXR, while the ubiquitination of proteins was simultaneously increased (30). Dysfunctions in the ubiquitin-proteasome pathway (UPP) have also been shown to play an important role in CVD (31). The overactivation of UPP has been identified as a contributing factor to the development of acute cardiotoxicity as an adverse event of the administration of anthracyclines.

\subsection{Enrichment analyses of hub genes}

The biological process, cellular composition, molecular function, and pathway of the target protein were analyzed by the WebGestalt database. The results obtained (Figure 3A) indicated that hub genes were significantly involved in a number of biological processes, including metabolic processes, 


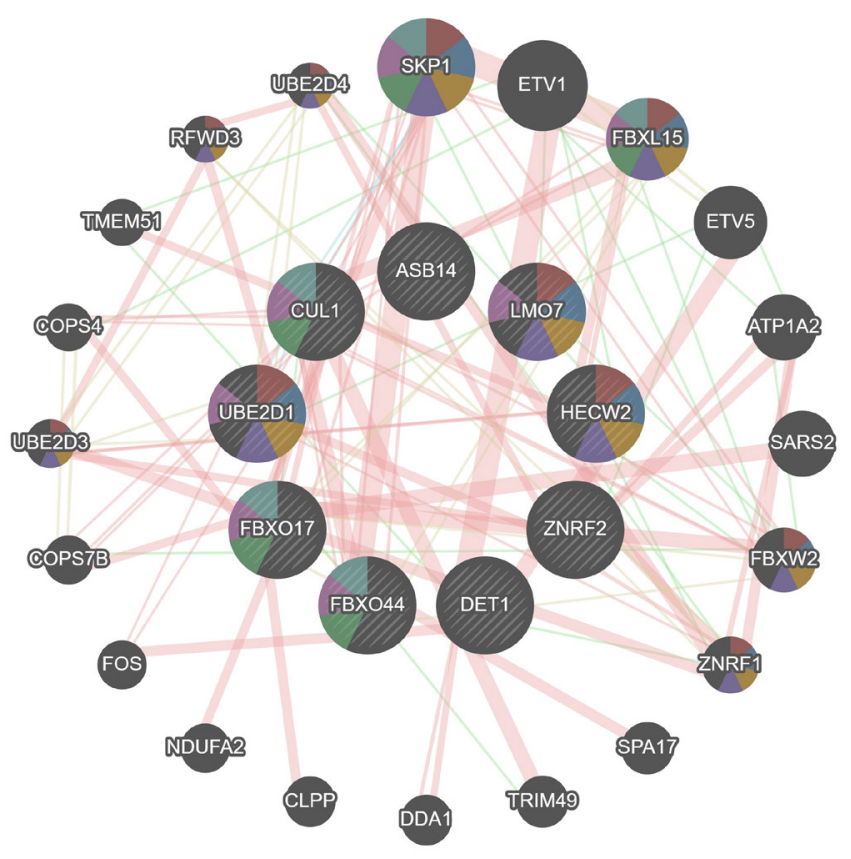

\section{Networks}

Physical Interactions $\quad 85.09 \%$

Shared protein domains $\quad 12.85 \%$

Pathway $\quad 1.80 \%$

Genetic Interactions $\quad 0.26 \%$

\section{Functions}

small conjugating protein ligase activity
ubiquitin-protein ligase activity
acid-amino acid ligase activity
ligase activity, forming carbon-nitrogen bonds
SCF ubiquitin ligase complex
ubiquitin ligase complex

Figure 4. Gene-gene interaction network among hub genes. A gene is represented by a node. The strength of the interaction is expressed by the size of the node. Inter-node connection lines represent the types of gene-gene interactions, while the line color shows the types of interactions. The possible function of each gene is represented by the color of the node.

biological regulation, cellular component organization, multicellular organismal processes, developmental processes, localization, cell communication, responses to stimuli, cell proliferation, and multi-organism processes. According to the classification of cellular components, the protein was located in a proteincontaining complex, the nucleus, and endomembrane system. The molecular function of the target protein involved transferase activity and protein binding. To further confirm that the biological processes involved in the treatment of myocardial injury by SFD play a role in the corresponding pathological events in the course of disease progression, we used the KEGG database for a pathway enrichment analysis. Seven important signal pathways were obtained $(p<0.05)$. These results (Figure $3 \mathrm{~B}$ ) indicated that the protective effects of SFD against myocardial injury involve seven signaling pathways. Previous studies demonstrated that SFD exerted cardiotonic effects by regulating the TNF signaling pathway (32), apoptosis (33), the PI3KAkt signaling pathway (34), TGF- $\beta$ /Smads signaling pathway (35), and Akt/eNOS signaling pathway (36). Ubiquitination (37) and neddylation (38) were also shown to play a role in myocardial injury; however, the relationships between SFD and these pathways need to be confirmed in further studies. Therefore, SFD appears to exert protective effects against myocardial injury by regulating 7 pathways and 10 biological processes. In addition, previous studies demonstrated that SFD may cure heart failure by regulating the TGF- $\beta /$ Smads signaling pathway, apoptosis, and the PI3K-Akt, Akt/
eNOS, and TNF signaling pathways. In summary, regarding myocardial injury, a number of proteins and pathways in the biological network may be regulated by SFD, which ultimately controls the occurrence and development of CVD.

\subsection{GeneMANIA analysis}

The GeneMANIA database was selected to further analyze the interaction network among hub genes (Figure 4). The 9 central nodes representing hub genes were surrounded by 20 nodes representing genes that strongly correlated with the hub genes in terms of physical interactions $(85.09 \%)$, shared protein domains $(12.85 \%)$, pathways $(1.8 \%)$, and genetic interactions $(0.26 \%)$. The top five genes displaying the strongest correlations with hub genes included S-phase kinaseassociated protein 1 (SKP1), ETS variant 1 (ETV1), F-box and leucine rich repeat protein 15 (FBXL15), ETV5, and ATPase $\mathrm{Na}^{+} / \mathrm{K}^{+}$transporting subunit alpha 2 (ATP1A2), among which SKP1 correlated with cullin 1 (CUL1), ubiquitin-conjugating enzyme E2D 1 (UBE2D1), F-box protein 17 (FBXO17), and FBXO44 in terms of physical interactions. ETV1 correlated with de-etiolated homolog 1 (DET1) in terms of physical interactions and with LIM domain only protein 7 (LMO7) and HECT with C2 and WW domain containing E3 ubiquitin protein ligase 2 (HECW2) in terms of genetic interactions. FBXL15 correlated with CUL1 and HECW2 in terms of physical interactions and with FBXO44 and FBXO17 in terms 
of shared protein domains. ETV5 correlated with DET1 in terms of physical interactions and with zinc and ring finger 2 (ZNRF2) and UBE2D1 in terms of genetic interactions. In addition, ATP1A2 correlated with ZNRF2 in terms of physical interactions. Further functional analyses revealed that these proteins showed the strongest correlation with ubiquitin-protein ligase activity (FDR $=4.45 \mathrm{E}-09)$. Additionally, these proteins correlated with small conjugating protein ligase activity, acid-amino acid ligase activity, ligase activity, the formation of carbon-nitrogen bonds, the Skp, Cullin, F-box containing complex (SCF) ubiquitin ligase complex, ubiquitin ligase complex, and cullin-RING ubiquitin ligase complex.

To simultaneously identify the target genes of SFD for protection against heart failure in the present study, the bioinformatic analysis method, including the identification of DEG, and a functional enrichment analysis were extensively used. A total of 1,134 DEG were identified using the STRING website. A module analysis showed that nine hub genes exhibited ubiquitin-protein ligase activity. In summary, the hub genes and related pathways discovered in the present study will provide a more detailed understanding of the mechanisms by which SFD protects the myocardium, which will lead to novel research concepts for SFD. Since the safety of drug treatments and the importance of communication with patients are increasing (39), the preventative and therapeutic effects of SFD on heart failure increase the safety of anticancer drug treatments and will also lead to peace of mind for patients.

\section{Funding: None.}

Conflict of Interest: The authors have no conflicts of interest to disclose.

\section{References}

1. Lloyd-Jones DM, Leip EP, Larson MG, d'Agostino RB, Beiser A, Wilson PW, Wolf PA, Levy D. Prediction of lifetime risk for cardiovascular disease by risk factor burden at 50 years of age. Circulation. 2006; 113:791-798.

2. Wang J, Qi F. Traditional Chinese medicine to treat COVID-19: the importance of evidence-based research. Drug Discov Ther. 2020; 14:149-150.

3. Kitazawa T, Park CH, Hiratani K, Choi JS, Yokozawa T. Efficacy of Chinese prescription Kangen-karyu for patient with metabolic syndrome. Drug Discov Ther. 2020; 14:5457.

4. Gu P, Chen H. Modern bioinformatics meets traditional Chinese medicine. Brief Bioinform. 2014; 15:984-1003.

5. Zhao J, Yang J, Tian S, Zhang W. A survey of web resources and tools for the study of TCM network pharmacology. Quant Biol. 2019; 7:17-29.

6. Fang J, Wang L, Wu T, Yang C, Gao L, Cai H, Liu J, Fang S, Chen Y, Tan W, Wang Q. Network pharmacology-based study on the mechanism of action for herbal medicines in Alzheimer treatment. J Ethnopharmacol. 2017; 196:281-
292.

7. Zhao J, Jiang P, Zhang W. Molecular networks for the study of TCM pharmacology. Brief Bioinform. 2010; 11:417-430.

8. Li B, Xu X, Wang X, Yu H, Li X, Tao W, Wang Y, Yang L. A systems biology approach to understanding the mechanisms of action of Chinese herbs for treatment of cardiovascular disease. Int J Mol Sci. 2012; 13:1350113520.

9. Yan X, Wu H, Ren J, Liu Y, Wang S, Yang J, Qin S, Wu D. Shenfu Formula reduces cardiomyocyte apoptosis in heart failure rats by regulating microRNAs. J Ethnopharmacol. 2018; 227:105-112.

10. Zheng SY, Sun J, Zhao X, Xu JG. Protective effect of shen-fu on myocardial ischemia-reperfusion injury in rats. Am J Chin Med. 2004; 32:209-220.

11. Yang D, Wang X, Wu Y, Lu B, Yuan A, Leon C, Guo N. Urinary metabolomic profiling reveals the effect of Shenfu Decoction on chronic heart failure in rats. Molecules. 2015; 20:11915-11929.

12. Chen Y, Yu R, Jiang L, Zhang Q, Li B, Liu H, Xu G. A comprehensive and rapid quality evaluation method of traditional Chinese medicine decoction by integrating UPLC-QTOF-MS and UFLC-QQQ-MS and its application. Molecules. 2019; 24:374.

13. He JL, Zhao JW, Ma ZC, Wang YG, Liang QD, Tan HL, Xiao CR, Tang XL, Gao Y. Serum pharmacochemistry analysis using UPLC-Q-TOF/MS after oral administration to rats of Shenfu Decoction. Evid Based Complement Alternat Med. 2015; 2015:973930.

14. Berger SI, Iyengar R. Network analyses in systems pharmacology. Bioinformatics. 2009; 25:2466-2472.

15. Zhou F, Hao G, Zhang J, Zheng Y, Wu X, Hao K, Niu F, Luo D, Sun Y, Wu L, Ye W, Wang G. Protective effect of 23-hydroxybetulinic acid on doxorubicin-induced cardiotoxicity: a correlation with the inhibition of carbonyl reductase-mediated metabolism. Br J Pharmacol. 2015; 172:5690-5703.

16. Szklarczyk D, Morris JH, Cook H, Kuhn M, Wyder S, Simonovic M, Santos A, Doncheva NT, Roth A, Bork P, Jensen LJ, von Mering C. The STRING database in 2017: quality-controlled protein-protein association networks, made broadly accessible. Nucleic Acids Res. 2017; 45:D362-D368.

17. Bader GD, Hogue CW. An automated method for finding molecular complexes in large protein interaction networks. BMC Bioinformatics. 2003; 4:2.

18. Liao Y, Wang J, Jaehnig EJ, Shi Z, Zhang B. WebGestalt 2019: gene set analysis toolkit with revamped UIs and APIs. Nucleic Acids Res. 2019; 47:W199-W205.

19. The Gene Ontology C. Expansion of the Gene Ontology knowledgebase and resources. Nucleic Acids Res. 2017; 45:D331-D338.

20. Kanehisa M, Furumichi M, Tanabe M, Sato Y, Morishima K. KEGG: new perspectives on genomes, pathways, diseases and drugs. Nucleic Acids Res. 2017; 45:D353-D361.

21. Warde-Farley D, Donaldson SL, Comes O, et al. The GeneMANIA prediction server: biological network integration for gene prioritization and predicting gene function. Nucleic Acids Res. 2010; 38:W214-220.

22. Li S, Zhang B, Jiang D, Wei Y, Zhang N. Herb network construction and co-module analysis for uncovering the combination rule of traditional Chinese herbal formulae. BMC Bioinformatics. 2010; 11 (Suppl 11):S6. 
23. Ding F, Zhang Q, Ung CO, Wang Y, Han Y, Hu Y, Qi J. An analysis of chemical ingredients network of Chinese herbal formulae for the treatment of coronary heart disease. PLoS One. 2015; 10:e0116441.

24. Guimera R, Nunes Amaral LA. Functional cartography of complex metabolic networks. Nature. 2005; 433:895900.

25. Wang X, Robbins J. Proteasomal and lysosomal protein degradation and heart disease. J Mol Cell Cardiol. 2014; 71:16-24.

26. Powell SR, Herrmann J, Lerman A, Patterson C, Wang $\mathrm{X}$. The ubiquitin-proteasome system and cardiovascular disease. Prog Mol Biol Transl Sci. 2012; 109:295-346.

27. Li YF, Wang X. The role of the proteasome in heart disease. Biochim Biophys Acta. 2011; 1809:141-149.

28. Minotti G, Menna P, Salvatorelli E, Cairo G, Gianni L. Anthracyclines: molecular advances and pharmacologic developments in antitumor activity and cardiotoxicity. Pharmacol Rev. 2004; 56:185-229.

29. Ranek MJ, Wang X. Activation of the ubiquitinproteasome system in doxorubicin cardiomyopathy. Curr Hypertens Rep. 2009; 11:389-395.

30. Sishi BJ, Loos B, van Rooyen J, Engelbrecht AM. Doxorubicin induces protein ubiquitination and inhibits proteasome activity during cardiotoxicity. Toxicology. 2013; 309:23-29.

31. Bulteau AL, Lundberg KC, Humphries KM, Sadek HA, Szweda PA, Friguet B, Szweda LI. Oxidative modification and inactivation of the proteasome during coronary occlusion/reperfusion. J Biol Chem. 2001; 276:3005730063.

32. Yu M, Ye L, Bian J, Ma L, Zheng C, Guo H. Effect of Jiawei Shenfu decoction on tumor necrosis factor-alpha and nuclear factor-kappa B in patients who have chronic heart failure with syndromes of deficiency of heart Yang. J Tradit Chin Med. 2019; 39:418-424.

33. Chen RJ, Rui QL, Wang Q, Tian F, Wu J, Kong XQ. Shenfu injection attenuates lipopolysaccharide-induced myocardial inflammation and apoptosis in rats. Chin J Nat Med. 2020; 18:226-233.
34. Zhu J, Song W, Xu S, Ma Y, Wei B, Wang H, Hua S. Shenfu injection promotes vasodilation by enhancing eNOS activity through the PI3K/Akt signaling pathway in vitro. Front Pharmacol. 2020; 11:121.

35. Ni J, Shi Y, Li L, Chen J, Li L, Li M, Zhu J, Zhu Y, Fan G. Cardioprotection against heart failure by Shenfu injection via TGF-beta/Smads signaling pathway. Evid Based Complement Alternat Med. 2017; 2017:7083016.

36. Wang YY, Li YY, Li L, Yang DL, Zhou K, Li YH. Protective effects of Shenfu injection against myocardial ischemia-reperfusion injury via activation of eNOS in rats. Biol Pharm Bull. 2018; 41:1406-1413.

37. Willis MS, Townley-Tilson WH, Kang EY, Homeister JW, Patterson C. Sent to destroy: the ubiquitin proteasome system regulates cell signaling and protein quality control in cardiovascular development and disease. Circ Res. 2010; 106:463-478.

38. Zou J, Ma W, Li J, Littlejohn R, Zhou H, Kim IM, Fulton DJR, Chen W, Weintraub NL, Zhou J, Su H. Neddylation mediates ventricular chamber maturation through repression of Hippo signaling. Proc Natl Acad Sci U S A. 2018; 115:E4101-E4110.

39. Yaguchi-Saito A, Yamamoto K, Sengoku T, Suka M, Sato T, Hinata M, Nakamura T, Nakayama T, Yamamoto M. Evaluation of rapid drug safety communication materials for patients in Japan. Drug Discov Ther. 2021; 15:101107.

Received August 3, 2021; Revised August 26, 2021; Accepted August 27, 2021.

*Address correspondence to:

Michihisa Tohda, Laboratory of Consilienceology for Wakanyaku, Section of Neuromedical Science, Institute of Natural Medicine, University of Toyama, Sugitani 2630, Toyama 9300194, Japan.

E-mail: tohdam@inm.u-toyama.ac.jp

Released online in J-STAGE as advance publication August 31, 2021. 